\title{
Quality of Movement and Spontaneous Use of The Affected Upper Limb In 8-Year-Old Infantile Hemiplegia After Applying Mirror Therapy. Case Series
}

\author{
Rocío Palomo Carrión* \\ Physiotherapy Department, CEU San Pablo University, Spain
}

*Corresponding author: Rocío Palomo Carrión PhD, Physiotherapy Department, CEU San Pablo University, Madrid, Spain.

Received Date: August 25, 2019

Published Date: August 30, 2019

\section{Abstract}

Introduction: Children with hemiplegia is a type of Cerebral Palsy where the function of the upper limb is disturbed, with limitation of function in it. The affected hand common characteristics are slowness, abnormal muscle tone, decreased strength and coordination difficulties. And to these problems are often added sensitivity alterations and mirror movements. One of the main problems of hemiplegia is the alteration of bimanual manipulation Based on the concept of mirror neurons, Mirror Therapy (MT) is applied to increase the affected upper limb function.

Objective: To assess the increase in the quality of movement and spontaneous use of the affected upper limb in congenital infantile hemiplegia with 8 years old after applying a Mirror Therapy protocol.

Materials and Methods: 4 children (8 years old) diagnosed with right congenital infantile hemiplegia are recruited to execute a Mirror Therapy protocol for 5 weeks, 15 minutes per day. Two measurements are carried out for the variables of quality of movement and spontaneous use of the affected upper limb.

Results: Increased 5\% for spontaneous use assessed with the Shriners Hospital Upper Extremity Evaluation, Shuee Evaluation and 6.5\% for the quality of movement measured through the Quality of Upper Extremity Skills Test, Quest scale, produced by a higher score in the final situation of the dissociated movements (increase 11\%).

Conclusion: Mirror Therapy performed in a time of 15 minutes per day for 5 weeks of treatment would allow improvements in spontaneous use and the quality of movement of the affected upper limb in children diagnosed with congenital infantile hemiplegia with an age of 8 years old.

Key words: Family; Mirror therapy; Physical therapy specialty; Infantile hemiplegia; House calls; Upper extremity

\section{Introduction}

Infantile Cerebral palsy (ICP) is one of the neurological disorders that most frequently causes disability in childhood and will persist in adulthood [1]. Its prevalence in developed countries remains stable, with around 2-3 cases per thousand live births [1,2].

Hemiplegia is a type of Cerebral Palsy where the function of the upper limb is disturbed, with limitation of function in it [3]. Ischemic or hemorrhagic strokes, brain tumors, congenital or traumatic brain injuries, and malformations are the most common causes of spastic hemiplegia in children [4].

The affected hand common characteristics are slowness, abnormal muscle tone, decreased strength and coordination difficulties. And to these problems are often added sensitivity alterations and mirror movements [5]. One of the main problems of hemiplegia is the alteration of bimanual manipulation [6]. The tendency of children with hemiplegia is to use the unaffected hand as dominant, even when the functional deficit is mild. The exclusion of this arm causes further deterioration including poor selective motor control, decreased range of motion, weakness, delayed skeletal maturation and increased muscle tone [7]. When the collaboration of the affected hand is required, the difficulties in bimanual tasks are more and more evident [5]. There are different arguments that address this problem. A study by Taub et al [8], associates this deterioration of the spontaneous use of the affected 
hand with the phenomenon of "no use learned". This means that the lack of use of the upper limb is not so much due to the neural damage itself, but to the custom of using the unaffected one to compensate.

Another factor related to bimanual activities is the presence of mirror movements. This occurs when repetitive and voluntary movements of one hand are accompanied by speculative and involuntary movements of the other hand $[6,7]$.

In a unilateral cerebral palsy (hemiplegia), the lesion interrupts normal crossed corticospinal projections of the injured hemisphere, but the other hemisphere preserves its ipsilateral corticospinal pathways. In this way, the hemisphere contrary to the injury can assume the motor control of the affected hand and both hands are controlled by the same hemisphere, resulting in the appearance of mirror movements [9]. This is a normal phenomenon at the beginning of motor development, but in children with hemiplegia increase and prolong over time [7]. These movements are present when using the affected hand to hold and stabilize objects during manipulation with the unaffected hand. This manipulation requires movements of the fingers, the hand and the arm, and when this is reflected in the static hand while holding the object, the grasp is hindered [9].

Kuhtz Buschbeck and his collaborators were the first to address the question of whether in a unilateral cerebral palsy the mirror movements interfered with bimanual coordination [10]. In the study it became clear that most patients whose affected hand was controlled by ipsilateral corticospinal projections, that is, most of those who have mirror movements, have a greater functional deficit of the affected hand compared to those who have preserved crossed corticospinal projections. However, this could justify why the affected hand is used less in bimanual tasks, without attributing a specific cause to mirror movements, but to the control of one hemisphere or another [10]. More specifically, Adler and colleagues [9] demonstrate that mirror movements have a significant impact on bimanual activities in children with hemiplegia. Cheads to a worse bimanual performance and a slower execution of activities. In addition, the deterioration of the affected hand in children with hemiplegia with mirror movements was more severe than in those who did not have mirror movements, which directly influences the bimanual function [9].

Giacomo Rizzolatti [11] directed a group of researchers who found a special type of visuomotor neurons. These neurons were in the ventral premotor area F5 of the premotor cortex of non-human primates, responsible for planning and executing the movements. The particularity of these neurons is that they not only activated during the execution of the action, but also did so during the observation of another individual performing similar actions [1113]. One of the distinguishing features of these neurons is that they are activated before movements in which the hand is directed to a target, such as being able to grasp an object or hold it [13].

Based on the concept of mirror neurons, Mirror Therapy (MT) is applied. The therapy was based on the use of a box with a mirror attached and located in the midline of the patient's trunk. The healthy upper limb was placed on one side of the box in such a way that it was reflected in the mirror. When the patient performed movements with his healthy upper limb and saw at the mirror from the side, he could see reflected symmetrical movements giving the sensation that his affected upper limb had a correct movement. After performing upper limb movements for a while and observing while the reflex of the healthy upper limb, many of the patients reported a strong improvement in their sensations. Ramachandran explains this phenomenon as a result of a lack of correspondence between the internal body image and the real body [14].

Unlike the evidence demonstrated by mirror therapy in the rehabilitation of the upper limb in adults, the evidence in children with hemiplegia is limited, since the starting points in both lesions are different [15]. Visual feedback with a mirror in children with hemiplegia obtained improvements in the active sense of joint position and grasp strength.

\section{Objective}

To assess the increase in the quality of movement and the spontaneous use of the affected upper limb in four children with 8 years old infantile hemiplegia.

\section{Material and Methods}

The ethical approval of the study was obtained by the ethical committee of the San Pablo CEU University. Before entering it, the informed consent of the parents or guardians of the children was acquired. Four subjects ( 2 girls and 2 boys, the 4 subjects with right hemiplegia) were obtained from the Virgen de la Salud Hospital following the established inclusion and exclusion criteria.

As inclusion criteria: diagnosis of congenital infantile hemiplegia, age 6-12 years, ability to grasp-release an object, level I-III of the Manual Ability Classification System (MACS) [16] and level I-III within Gross Motor Function Classification System (GMFCS) [17]. The exclusion criteria would be: Diseases not associated with congenital infantile hemiplegia, low cognitive level, presence of contractures, surgery 6 months prior to the intervention, botulinum toxin 2 months prior to or during the intervention, and pharmacologically uncontrolled epilepsy.

The 4 children were diagnosed with congenital infantile hemiparesis with a mean age of 8 years, being in a level II within the MACS and a level I within the GMFCS. The 4 subjects completed the intervention protocol successfully, with no abandonment by any family.

\section{Design and procedure}

The results of the different variables were measured in 2 stages: at the beginning of the treatment, and at the end of the treatment (5 weeks).

\section{Interventions}

Home intervention was designed, carried out in a routine of 15 minutes per day for a period of 5 weeks. 
4 exercises were scheduled: 1-Grasp a small ball; 2-Distal grasp, 3-Extension of the wrist joint, 4-Forearm supination. Each exercise was performed for 3 minutes and one minute was rested between exercises, thus completing the structured time of 15 minutes per day of mirror therapy (Figure 1).

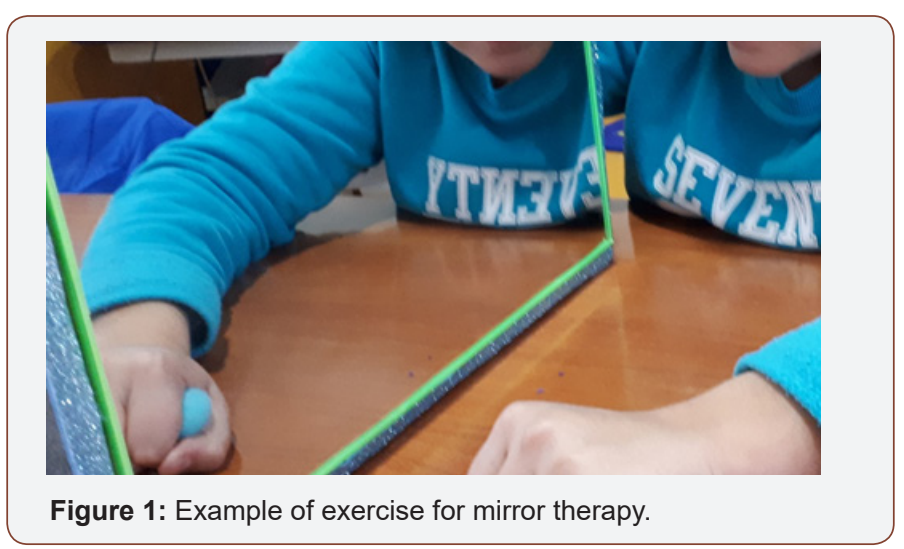

Each of the families was instructed to make their own mirror box and decorate it according to the children's interests (Figure 2).

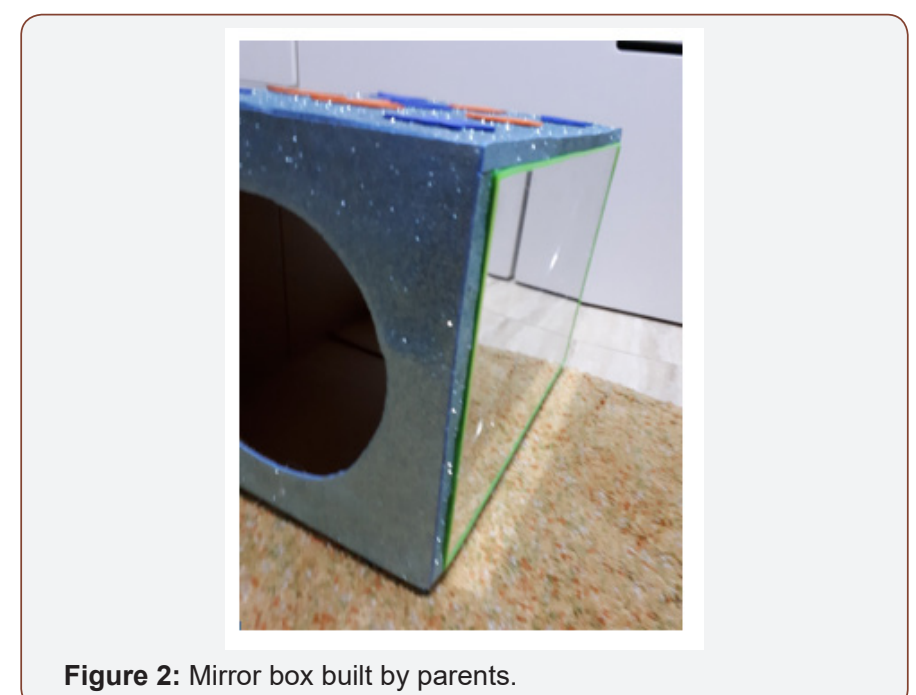

The therapy was realized by family at home. The parents had an instruction prior to the beginning of the intervention where they were taught how to do the activities, as well as they had to control the time to execute them. Once the intervention was clear, the parents were asked for daily videos of the therapy to ensure its correct execution. In addition, to write down each day if they had spent time, existence of problems at the time of executing any of the exercises, how was the child's tolerance, and the time allocated to each exercise to see throughout the week. The therapist was followed in person by the therapist to accompany the parents during the therapy, as well as to clarify the pertinent doubts and make corrections if necessary.

\section{Primary measures}

Spontaneous use was assessed with the Shriners Hospital Upper Extremity Evaluation, Shuee Evaluation [19], which was used to record the values in the two measurements. This evaluation is based on the recording of videos of children performing a series of tasks to observe the hand functionality and the alignment of the joint segments of the affected upper limb in hemiplegic children in an age between 3 and 18 years. Spontaneous use is valued depending on the non-participation or active participation of affected upper limb in the execution of 9 tasks: 1- Remove bills from a wallet, 2- Fold a paper, 3- Tear a paper, 4- Thread beads of a necklace, 5- Unscrew a cap from a bottle, 6- Separate plasticine with your hands, 7- Cut plasticine with a knife, 8- Put on your socks, 9- Tying the laces.

The modified House scale is used for scoring tasks with a scale from 1 to 5 , corresponding to 1 to $0 \%$ understood as the non-use of the affected limb in the task and the value 5 takes the value of $100 \%$ for the existence of spontaneous use from partial to complete.

\section{Secondary measures}

Quality of movement and bimanual dexterity were measured through the Quality of Upper Extremity Skills Test, Quest scale [20]. It gives us a numerical value that is obtained from the average of the percentages in 36 items distributed in four categories: dissociated movements, grasp, supports and postural reactions of both upper limbs. It takes a value from 0 to 100 expressed in percentages. $100 \%$ being the maximum quality value of both upper extremities, measuring each upper limb separately but without making a distinction between healthy and affected upper limb in the final percentage of each category. It was designed for children with neuromotor dysfunction with spasticity, in order to assess the quality of manual function in children with cerebral palsy. It is validated for children between 18 months and 8 years of age [20].

\section{Data analysis}

A descriptive analysis of the data obtained from each of the children was carried out for the different variables, based on the mean value to execute the comparisons between them.

\section{Results and Discussion}

Table 1 shows the mean data obtained for the two measurements taken of the studied variables in the different subjects.

Table 1: Mean values of the Quality of Movement and Spontaneous use for the baseline and final situation of the studied variables in the 4 subjects.

\begin{tabular}{|c|c|c|}
\hline Studied Variable & $\begin{array}{c}\text { Pre-Treatment } \\
\text { (Assessment) }\end{array}$ & $\begin{array}{c}\text { Post-Treatment } \\
\text { (Assessment) }\end{array}$ \\
\hline \multicolumn{3}{|c|}{ SHUEE Scale } \\
\hline Spontaneous Use & $53 \%$ & $58 \%$ \\
\hline Quality of Movement & $67.50 \%$ & $74 \%$ \\
\hline Dissociated Movements & $58 \%$ & $69 \%$ \\
\hline Grasp & $67 \%$ & $72 \%$ \\
\hline Supports & $70 \%$ & $75 \%$ \\
\hline Postural Reactions & $75 \%$ & $80 \%$ \\
\hline
\end{tabular}

During the execution of the intervention, an increase can be observed for all the variables, being $5 \%$ for spontaneous use and $6.5 \%$ for the quality of movement, produced by a higher score in the final situation of the dissociated movements (increase 11\%) as can be seen in Figure 3. 


\section{Spontaneous Use and Quality of Movement}

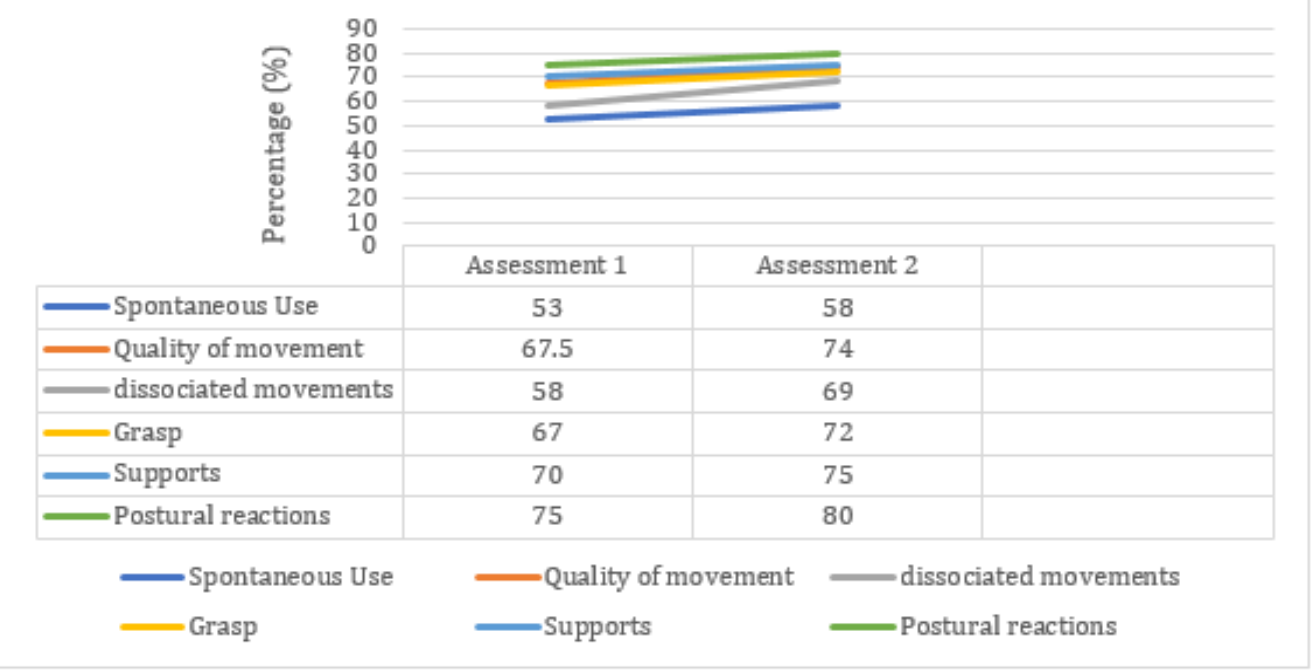

Figure 3: Increase in pre-post intervention values for movement quality and spontaneous use.

Once the results were assessed, it may think that the 4 children who performed the intervention obtained improvements in both variables at the end of the intervention, suggesting that the therapy was successfully performed at home and as shown in the records and in the follow-up carried out it was possible to verify that the proposed times had been fulfilled. This indicates that such therapy is effective in the short term.

Improves spontaneous use (5\%) allow children able to stably hold the object with the affected hand and manipulate it with the healthy one. Concept that previously in some occasions the children did not have a great stability in the grasp, and They executed the stabilization with the affected forearm instead of using the hand for it. In the study of Gygax et al [21], Mirror Therapy was applied in children from 6 to 14 years old with a level of MACS I-II. The protocol was done for 3 weeks, 15 minutes per day in which the spontaneous use of the affected upper limb was also measured, obtaining an increase of $2.7 \%$, being lower than the increase in the study presented, which suggests that the 5 weeks of intervention (in our study) could allow greater improvements in the active participation of the affected upper limb when performing bimanual tasks (spontaneous use) as well as be done at home can be an initiative to take into account since parents can be involved in carrying out the activities and observing the limitations present in the affected upper limb in their children.

There is difficulty in comparing the results obtained for the quality of movement since most studies use other tools such as the modified Melbourne Assessment (MA2) so it is difficult to make the comparison between them $\neg 22$. In our study we observed an increase in the total movement quality produced by the improvement in dissociated movements, which suggests that Mirror Therapy allows the child with hemiplegia who has difficulties in executing the selectivity of movements of his affected upper limb, and the Child would acquire a greater variability of movements when performing Mirror Therapy. This greater selectivity of movements also translates into an improvement in the grasp, support and postural reactions valued in our study, which would subsequently facilitate spontaneous use and would improve the quality of execution of bimanual tasks.

\section{Limitations of The Study and Future Research Lines}

The study sample was small, so the results cannot be generalized to the congenital infantile hemiplegia population. It would be necessary to expand the sample and have a control group to demonstrate the effectiveness of the therapy. As well as being able to combine it with other therapies of scientific evidence that improve the spontaneous use of the affected upper limb such as modified Constraint Induced Movement Therapy (CIMT) or Bimanual Intensive Therapy (BIT).

\section{Conclusion}

Mirror Therapy performed in a time of 15 minutes per day for 5 weeks of treatment would allow improvements in spontaneous use and the quality of movement of the affected upper limb in children diagnosed with congenital infantile hemiplegia with an age of 8 years old.

\section{Acknowledgement}

Thank the families and children who did the combined intensive protocol because Their work was excellent and met the times an all the activities Proposed successfully.

\section{Conflict of Interest}

There is not conflict of interest by the author.

\section{References}

1. Bleyenheuft Y, Gordon AM (2013) Precision grip control, sensory impairments and their interactions in children with hemiplegic cerebral palsy: a systematic review. Res Dev Disabil 34(9): 3014-3028.

2. Rosenbaum P, Paneth N, Leviton A, Goldstein M, Bax M, et al. (2007) A report: the definition and classification of cerebral palsy April 2006. Dev Med Child Neurol Suppl 109: 8-14. 
3. Holsbeeke L, Ketelaar M, Schoemaker MM, Gorter JW (2009) Capacity, capability, and performance: different constructs or three of a kind? Arch Phys Med Rehabil 90(5): 849-855.

4. Boyd RN, Ziviani J, Sakzewski L, Novak I, Badawi N, et al. (2017) REACH: study protocol of a randomised trial of rehabilitation very early in congenital hemiplegia. BMJ Open 7(9): e017204.

5. Holmefur M, Krumlinde-Sundholm L, Bergström J, Eliasson A (2010) Longitudinal development of hand function in children with unilateral cerebral palsy. Dev Med Child Neurol 52(4): 352-357.

6. Fedrizzi E, Pagliano E, Andreucci E, Oleari G (2003) Hand function in children with hemiplegic cerebral palsy: prospective follow-up and functional outcome in adolescence. Dev Med Child Neurol 45(2): 85-91.

7. Hoare B, Imms C, Carey L, Wasiak J (2007) Constraint-induced movement therapy in the treatment of the upper limb in children with hemiplegic cerebral palsy: a Cochrane systematic review. Clin Rehabil 21(8): 675685.

8. Taub E, Ramey SL, DeLuca S, Echols K (2004) Efficacy of constraintinduced movement therapy for children with cerebral palsy with asymmetric motor impairment. Pediatrics 113(2): 305-312.

9. Adler C, Berweck S, Lidzba K, Becher T, Staudt M (2015) Mirror movements in unilateral spastic cerebral palsy: Specific negative impact on bimanual activities of daily living. Eur J Paediatr Neurol 19(5): 504509.

10. Kuhtz-Buschbeck JP, Sundholm LK, Eliasson AC, Forssberg H (2000) Quantitative assessment of mirror movements in children and adolescents with hemiplegic cerebral palsy. Dev Med Child Neurol 42(11): 728-736.

11. Rizzolatti G, Fadiga L, Gallese V, Fogassi L (1996) Premotor cortex and the recognition of motor actions. Brain Res Cogn Brain Res 3(2): 131141.

12. Rizzolatti G, Fogassi L (2014) The mirror mechanism: recent findings and perspectives. Philos Trans R Soc Lond B Biol Sci 369(1644): 20130420.
13. Mukamel R, Ekstrom AD, Kaplan J, Iacoboni M, Fried I (2010) Singleneuron responses in humans during execution and observation of actions. Curr Biol 20(8): 750-756.

14. Guenther K (2016) 'It's All Done With Mirrors': V.S. Ramachandran and the Material Culture of Phantom Limb Research. Med Hist 60(3): 342358.

15. Weisstanner C, Saxer S, Wiest R, Kaelin-Lang A, Newman CJ, et al. (2017) The neuronal correlates of mirror illusion in children with spastic hemiparesis: a study with functional magnetic resonance imaging. Swiss Med Wkly 147: w14415.

16. Bruchez R, Jequier Gygax M, Roches S, Fluss J, Jacquier D, et al. (2016) Mirror therapy in children with hemiparesis: a randomized observerblinded trial. Dev Med Child Neurol 58(9): 970-978.

17. Eliasson AC, Krumlinde-Sundholm L, Rösblad B, Beckung E, Arner M, et al. (2006) The Manual Ability Classification System (MACS) for children withcerebral palsy: scale development and evidence of validity and reliability. Dev Med Child Neurol 48(7): 549-554.

18. Palisano R, Rosenbaum P, Walter S, Russell D, Wood E, et al. (1997) Development and reliability of a system to classify gross motor function in children with cerebral palsy. Dev Med Child Neurol 39(4): 214-223.

19. Davids JR, Peace LC, Wagner LV, Gidewall MA, Blackhurst DW, et al. (2006) Validation of the Shriners Hospital for Children Upper Extremity Evaluation (SHUEE) for children with hemiplegic cerebral palsy. J Bone Joint Surg Am 88(2): 326-333.

20. Thorley M, Lannin N, Cusick A, Novak I, Boyd R (2012) Construct validity of the Quality of Upper Extremity Skills Test for children with cerebral palsy. Dev Med Child Neurol 54(11): 1037-1043.

21. Gygax MJ, Schneider P, Newman CJ (2011) Mirror therapy in children with hemiplegia: a pilot study. Dev Med Child Neurol 53(5): 473-476.

22. Park EJ, Baek SH, Park S (2016) Systematic review of the effects of mirror therapy in children with cerebral palsy. J Phys Ther Sci 28(11): 3227-3231. 